\title{
Mechanism of Hb F Stimulation by S-Stage Compounds \\ In Vitro Studies with Bone Marrow Cells Exposed to 5-Azacytidine, Ara-C, or Hydroxyurea
}

\author{
R. Galanello, G. Stamatoyannopoulos, and Th. Papayannopoulou \\ Divisions of Hematology and Medical Genetics, Department of Medicine, University of Washington, Seattle, Washington 98195
}

\begin{abstract}
The in vitro effect of S-stage-specific drugs on the fetal hemoglobin (Hb F) potential of erythroid precursors and progenitors was tested by exposing bone marrow cells to 5-aza-2'-deoxycytidine, Ara-C, or hydroxyurea in suspension cultures and reculturing the cells in drug-free clonal cultures. Analysis of Hb F in the erythroblasts present at the end of suspension cultures and in the erythroid colonies formed from treated progenitors showed that $1 \times 10^{-9}-5 \times 10^{-8}$ M 5-aza-2'-deoxycytidine produced a concentration-related increase in the proportion of $\mathrm{Hb}$ F-positive erythroblasts, of $\mathrm{Hb}$ F-positive erythroid CFU (CFUe) colonies, and at the higher doses used, an increased Hb F expression in erythroid burst-forming unit (BFUe)-derived colonies. Preincubation of bone marrow cells with Ara-C produced significant megaloblastic changes by the end of the 2-d incubation and increased the proportion of $\mathbf{H b}$ F-positive erythroblasts, CFUe colonies, and e-clusters, but BFUe-derived progeny was unaffected. Hydroxyurea failed to produce significant changes in $\mathrm{Hb} F$ at the range of concentrations used. The data raise the possibility of more than one mechanism underlying the stimulation of $\mathrm{Hb} F$ by S-stage drugs.
\end{abstract}

\section{Introduction}

Recently, several pharmacologic agents have been used to stimulate fetal hemoglobin $(\mathrm{Hb} \mathrm{F})^{1}$ synthesis in vivo. The initial studies involved treatment of baboons (1) or patients with either Cooley's anemia (2-4) or sickle cell anemia (3-7) with 5 -azacytidine. The animals or the patients responded with considerable stimulation of $\gamma$-chain synthesis, which was initially attributed to the hypomethylation of $\gamma$-globin genes (1-6). Subsequent studies (8-14), however, called attention to the fact that the drug treatments triggered acute-phase regeneration kinetics, previously known to be associated with heightened $\mathrm{Hb} F$ formation in vivo $(15,16)$, and suggested that stimulation of $\mathrm{Hb}$ F synthesis by 5-azacytidine, as well as by other compounds is mainly indirect and cell kinetic in origin (8-14). The contribution of this mechanism to the in vivo

Address correspondence to Dr. Papayannopoulou, Dept. of Medicine, Div. of Hematology, RM-10, University of Washington, Seattle, WA 98195.

Received for publication 16 March 1987 and in revised form 19 October 1987.

1. Abbreviations used in this paper: BFUe, erythroid BFU; CFUe, erythroid CFU; e-cluster, erythroid cluster; $\mathrm{Hb}$ F, fetal hemoglobin.

J. Clin. Invest.

(C) The American Society for Clinical Investigation, Inc.

0021-9738/88/04/1209/08 \$2.00

Volume 81, April 1988, 1209-1216 stimulation of $\mathrm{Hb} \mathrm{F}$ by 5-azacytidine or hydroxyurea has, however, been questioned by other investigators $(6,17)$.

To investigate further the mechanisms of induction of $\mathrm{Hb}$ F by cell cycle-specific drugs, we applied an experimental system consisting of exposure of bone marrow cells to S-stage compounds for 42-60 h in suspension culture, followed by cloning of these cells in a drug-free environment. This system allows testing of in vitro induction of $\mathrm{Hb} \mathrm{F}$ by a drug and, in addition, permits an assessment of the level of erythroid cell development on which the effect of the drug is exerted and of associated changes in proliferation/maturation parameters. We find, by a variety of approaches, that 5-aza-2'-deoxycytidine increased $\mathrm{Hb} \mathrm{F}$ in erythroblasts, erythroid clusters (eclusters), erythroid CFU (CFUe), and erythroid burst-forming unit (BFUe) colonies, while Ara-C stimulates $\mathrm{Hb}$ F expression in CFUe colonies, e-clusters, and erythroblasts. We find no such effects in the concentrations of hydroxyurea we used. The mechanism by which these compounds exert their in vitro effect could be direct (i.e., demethylation of $\boldsymbol{\gamma}$-globin genes) or indirect, through changes in the kinetics of in vitro maturation (i.e., megaloblastosis), or through progenitor cell selection and effect on the rate of their cell cycling. The data raise the possibility that these drugs, in addition to their triggering of acute regeneration kinetics in vivo, can have other effects, which may account for the different levels of $\mathrm{Hb} F$ achieved in vivo, or the rapidity of $\mathrm{Hb} \mathrm{F}$-reticulocyte response in some patients.

\section{Methods}

Experimental approach. After density separation of aspirated bone marrow cells, the mononuclear interphase cells were washed and incubated in the media described below (see suspension cultures). Experimental samples contained the compound under testing, while controls contained media without that compound. After 42 (and in some experiments, after 60 ) $\mathrm{h}$ of incubation, the samples were replated in semi-solid media (plasma clots or methylcellulose) in the absence of the drug. Colonies were counted in these clonal cultures, and $\mathrm{Hb} F$ was analyzed, as described later. With this approach, we tested whether preexposure, in the suspension culture, of bone marrow erythroid progenitors for 42-60 h to 5-aza-2'-deoxycytidine, to Ara-C, or to hydroxyurea influences $\mathrm{Hb} \mathrm{F}$ expression in the erythroid colonies produced by these progenitors in clonal cultures done in a drug-free environment. To examine whether Ara-C or 5-aza-2'-deoxycytidine influenced $\mathrm{Hb} \mathrm{F}$ expression on the morphologically recognizable (at the end of suspension culture) erythroid cells, an aliquot from the suspension culture was used for erythroblast purification (see "panning") and $\mathrm{Hb} \mathrm{F}$ expression in these erythroblasts was assessed after labeling with anti- $\beta$ and anti- $\gamma$-chain antibodies. In the present studies, 5-aza-2'-deoxycytidine was used instead of 5-azacytidine because of its higher potency and lack of incorporation into RNA (18). In addition, for the clonal cultures, instead of FCS, we have used a treated serum preparation which largely abrogates $\mathrm{Hb} F$ activation in vitro (unpublished data).

Suspension cultures. Mononuclear cells were isolated after layering the samples on a cushion of Ficoll and diatrizoate salts (LSM, Bionetics 
Laboratory Products, Litton Bionetics, Inc., Charleston, SC) at a density of $1.077-1.080 \mathrm{~g} / \mathrm{ml}$ and centrifugation at $400 \mathrm{~g}$ for $40 \mathrm{~min}$ at $22^{\circ} \mathrm{C}$.

Suspension culture media consisted of $20 \%$ FCS (Armour Pharmaceutical, Kankakee, IL), 1\% BSA (Sigma Chemical Co., St. Louis, MO), erythropoietin (Terry Fox Laboratory, Vancouver, British Columbia, Canada) $(0.5 \mathrm{IU} / \mathrm{ml})$, and the desired concentration of the compound under testing. Bone marrow mononuclear cells suspended in the above media at a final concentration of $5 \times 10^{6}$ cells $/ \mathrm{ml}$ were incubated at $37^{\circ} \mathrm{C}$ in a highly humidified $\mathrm{CO}_{2}$ incubator for $42-60 \mathrm{~h}$.

The three compounds under study, 5-aza-2'-deoxycytidine (Sigma Chemical Co.), Ara-C (Upjohn Co., Kalamazoo, MI), or hydroxyurea (Sigma Chemical Co.) were dissolved immediately before their use in the suspension cultures. Thereafter, every $12 \mathrm{~h}$ the cells were spun, and most of the supernate media were removed and replaced by an equal volume of fresh medium, to which newly dissolved drug was added.

Immune adherence (panning). For immune adherence, previously described methodology (19) was adopted. Briefly, after suspension culture, the cells were washed, resuspended in PBS containing $2.5 \%$ FCS, and the cell suspension was gently added to a polystyrene sterile petri dish previously coated with a 1:200 dilution (in $0.05 \mathrm{M}$ Tris, $\mathrm{pH}$ 9.5) of monoclonal antibody Ep-1, an antibody specific for erythroid progenitors and erythroblasts (20). After $70 \mathrm{~min}$ incubation at $4^{\circ} \mathrm{C}$ on a level surface, the plates were washed three times with cold PBS containing $1 \%$ FCS, and the nonadherent cells were aspirated. The adherent cells were recovered by pipetting vigorously the entire surface of the plate with PBS plus $1 \%$ FCS. The so-recovered adherent cells were $>97 \%$ erythroid. We have previously shown by cell sorting and complement-dependent cytotoxicity experiments (20) that Ep-1 antigen is present in most of the BFUe, in virtually all CFUe, and in all erythroblasts. Therefore, apart from erythroblasts, erythroid progenitors (mainly CFUe) are also present in these Ep-1-adherent preparations, but their numbers are low compared with the population of erythroid precursors (unpublished data). Detached cells from Ep-1coated plates were used for smear preparation. Smears were either stained with Wright-Giemsa or they were labeled with fluorescent antiglobin chain monoclonal antibodies.

Clonal cultures. Bone marrow cells from the suspension cultures were washed and inoculated $\left(1-3 \times 10^{5}\right.$ cells $\left./ \mathrm{ml}\right)$ in methylcellulose and plasma clot supporting media using previously described methods $(21,22)$. After 3-4 d in culture, plasma clot preparations were stained with benzidine for colony counting, or they were doubly labeled with fluorescent antiglobin $(\gamma$ and $\beta$ ) monoclonal antibodies for analysis of $\mathrm{Hb} \mathrm{F}$ expression. e-clusters were aggregates of cells composed of two to seven well-hemoglobinized cells. CFUe-derived colonies were those comprised of 8 to 64 well-hemoglobinized cells. Colonies derived from BFUe were counted on day 12 to 14 methylcellulose cultures.

Analysis of cells and colonies by immunofluorescence. Cytocentrifuge smears of erythroid cells enriched by immune adherence were air dried, fixed in methanol for $18 \mathrm{~min}$, rinsed in PBS, and subsequently in distilled water. The areas containing the fixed cells were overlaid with a few microliters of anti- $\gamma$ monoclonal antibody and incubated for $1 \mathrm{~h}$ at $37^{\circ} \mathrm{C}$ in a humidifed chamber. After washing in PBS and distilled water, the preparations were dried, covered with anti-mouse IgG $\mathrm{F}\left(\mathrm{ab}^{\prime}\right)_{2}$ conjugated to FITC (Tago Inc., Burlingame, CA), and incubated, washed, and dried, as described above. The cells were then doubly labeled with an anti- $\beta^{\wedge}$-globin monoclonal (or, when indicated, with anti- $\beta^{\mathbf{S}}$ monospecific) antibody directly conjugated to rhodamine. After incubation for $1 \mathrm{~h}$ at $37^{\circ} \mathrm{C}$ in a humidified chamber, the preparations were washed and dried. The doubly labeled preparations were viewed in a Zeiss Universal fluorescent microscope with the aid of appropriate filters. To assess $\mathrm{Hb} \mathrm{F}$ expression in erythroid colonies, flattened plasma clots were fixed and labeled with the anti- $\beta$ and the anti- $\gamma$-chain antibodies, as above.

Erythroblasts were considered to be $F$ positive if they were labeled with anti- $\gamma$-chain FITC. They were $F$ negative if they were labeled only by the anti- $\beta^{\mathrm{A}}$ (or the anti- $\beta^{\mathrm{S}}$ ) antibody. The e-clusters and CFUe colonies were considered as A positive when all the cells were $\beta$-glo- bin-positive and $\gamma$-negative; they were considered as $F$ positive when two or more cells of a colony were $\gamma$-globin-positive, while all the cells of the colony were $\beta$ positive. (Colonies containing only one F-positive cell were very few, and they were not included since, occasionally, it was not clear whether a single F-positive erythroblast was part of a single colony or represented superimposition of a free background erythroblast.) Colonies that appeared "young" (i.e., few, large cells) and were negative in $\mathrm{Hb} \mathrm{A}$ were not considered as CFUe. Frequencies of F-positive e-clusters or F-positive CFUe were calculated after counting at least 50 (range from 50 to over 500 ) colonies in each category. All counting was done by one person. Measurements of $F$ colonies from multiple clots inoculated with the same cells have shown that the fluorescent method of $F$ analysis has $<5 \%$ experimental error.

The sensitivity of the fluorescent antibody detection method has been assessed with previous experiments. Studies of cells of known hemoglobin content (23) or with known proportion of an abnormal hemoglobin fraction (24) indicate that fluorescent anti-globin chain antibodies can detect at least $0.1 \mathrm{pg}$ of hemoglobin per red cell.

Globin biosynthesis. For measuring $\mathrm{Hb} F$ production in e-clusters and CFUe colonies, 6 to 14 clots (depending on the number of colonies per clot) were incubated at $37^{\circ} \mathrm{C}$ for $6 \mathrm{~h}$ in the presence of leucine-free media, $10 \% \mathrm{FCS}$, and $100 \mu \mathrm{Ci} / \mathrm{ml}$ of $\left[{ }^{3} \mathrm{H}\right]$ leucine. At the end of the incubation, cells were lysed, and hemoglobin was extracted by centrifugation at $8,000 \mathrm{rpm}$. For measuring globin biosynthesis in BFUe colonies, fully hemoglobinized erythroid bursts were lifted from the plates, pooled, and incubated with $\left[{ }^{3} \mathrm{H}\right]$ leucine $(100-200 \mu \mathrm{Ci} / \mathrm{ml})$ in leucine-free medium. Cell lysates were processed for isoelectric focusing of globin chains in the presence of NP40, $\beta$-mercaptoethanol, and 8 $M$ urea, as described before (25). The relative synthesis of globin chains was estimated from densitometric tracings of the resultant fluorograms.

Analysis of data. Numbers of erythroid progenitors recovered after suspension culture and frequencies of F-positive colonies at various concentrations of cell cycle-specific drugs are presented in Table I. To correct for the variation between experimental samples (which is especially profound in the case of clonogenic assays; see Table I), the results in each drug concentration were expressed as percent of the values obtained when the same cells were cultured in the absence of cell cycle-specific drugs. Linear regression analysis was used to test the statistical significance of the results.

\section{Results}

Effects of 5-aza-2'-deoxycytidine. Bone marrow cells from normal volunteers were cultured in suspension, either in the absence of 5-aza-2'-deoxycytidine (controls) or in the presence of a given concentration of this compound. At the end of the 42-h incubation period, the proportion of viable cells in the control and in the 5-aza-2'-deoxycytidine-treated cultures was 74.3 and $67.6 \%$ (respectively) of the inoculated cells. After the incubation in the suspension cultures, the bone marrow cells were inoculatd in plasma clot or methylcellulose cultures (done in the absence of 5-aza-2'-deoxycytidine), and numbers of erythroid colonies, as well as $\mathrm{Hb} \mathrm{F}$ expression, were measured.

As shown in Table I, incubation with 5-aza-2'-deoxycytidine affected the clonogenic potentials of erythroid progenitors. While there was only a minor decrease in e-clusters and CFUe when $1 \times 10^{-8}-1 \times 10^{-9}$ M 5-aza-2'-deoxycytidine were present in culture, at $5 \times 10^{-8} \mathrm{M}$, erythroid progenitors were reduced to $10-43 \%$ of control values (Fig. 1). No colonies were grown in the clonal cultures when the bone marrow cells had been exposed to $1 \times 10^{-6} \mathrm{M}$ 5-aza-2'-deoxycytidine during the suspension culture.

The effect of 5-aza-2'-deoxycytidine on $\mathrm{Hb} \mathrm{F}$ expression in e-clusters and, separately, in CFUe colonies was assessed by 
Table I. Erythroid Progenitors in Bone Marrow Cells Cultured in Suspension for $42 h^{*}$ in the Presence or Absence of Cell Cycle-specific Compound

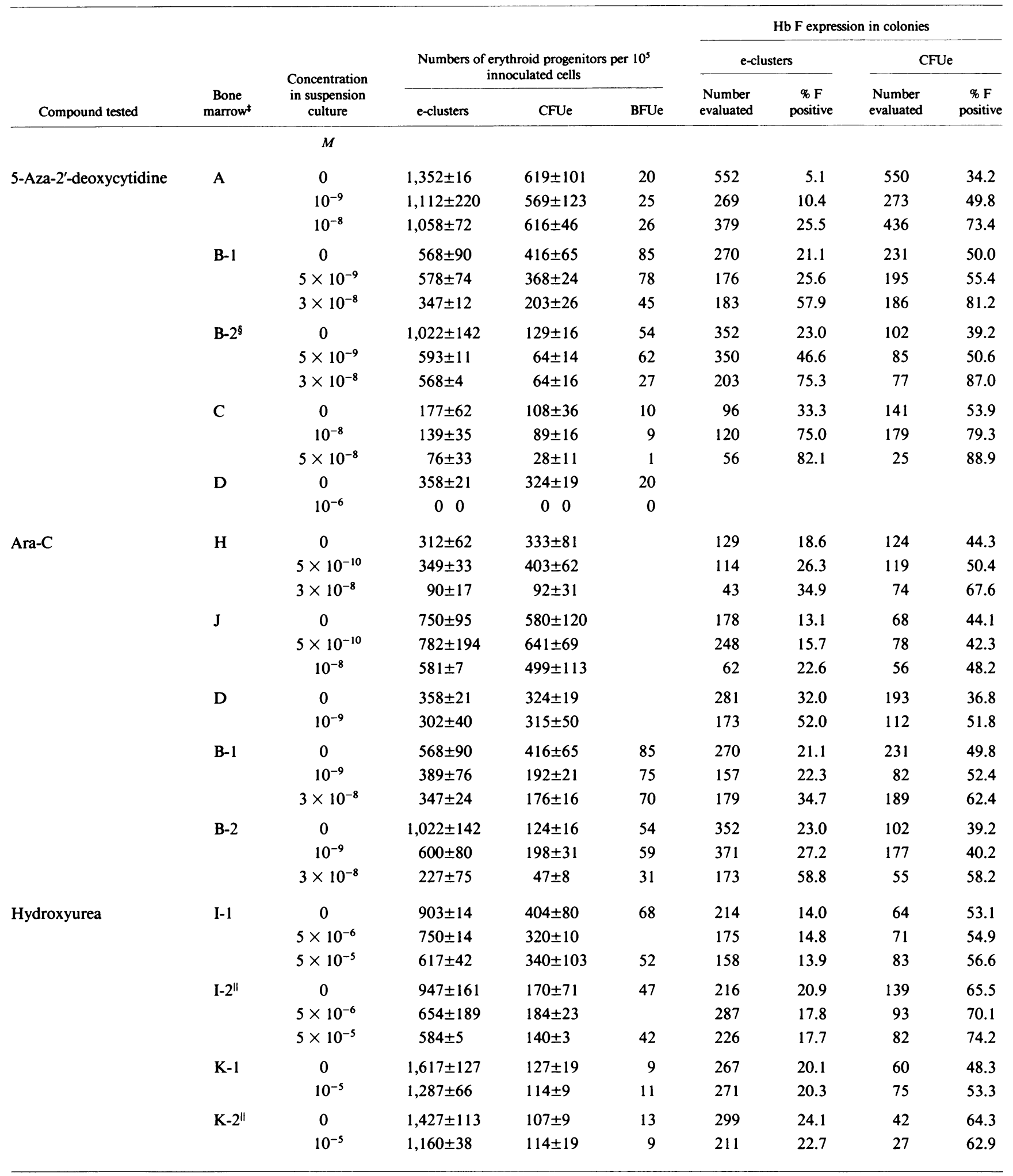

* Except for bone marrow B-2. ${ }^{\ddagger}$ All bone marrow donors were persons without a hemoglobinopathy, $<1 \% \mathrm{Hb}$ F, and $<2-5 \% \mathrm{~F}$ cells. ${ }^{\S}$ The bone marrow suspension culture lasted for $60 \mathrm{~h}$ (see text). "I-2 and K-2 were cultured in the presence of untreated FCS. All other bone marrow cultures were done with charcoal-treated FCS. 

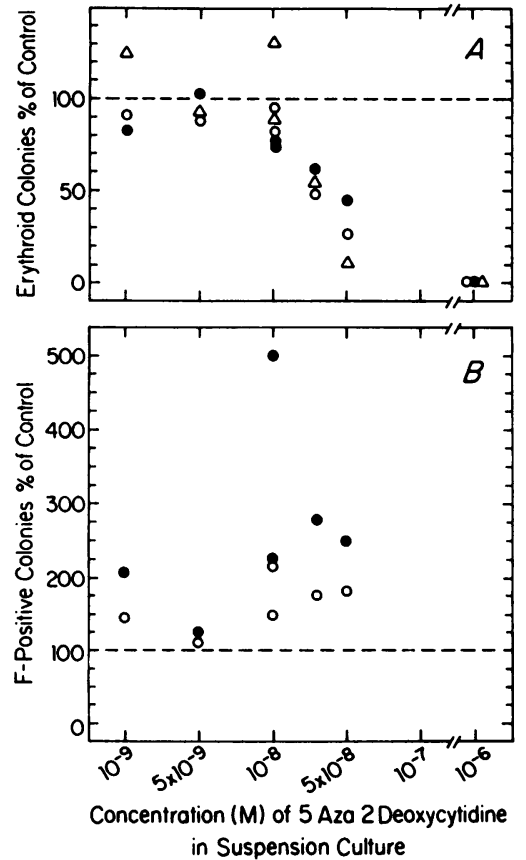

in Suspension Culture

the suspension cultures) in the absence of text). Findings in (๑) e-clusters, ( 0 ) CFUe colonies, and $(\Delta)$ BFUe colonies.

labeling the plasma clot preparations with anti- $\beta$ and anti- $\gamma$ chain monoclonal antibodies and analyzing the colonies as described in Methods. In the control samples, the range of $\mathrm{Hb}$ $\mathrm{F}$-positive e-clusters was from 5 to $35 \%$ and of $\mathrm{Hb} \mathrm{F}$-positive CFUe from 34 to 55\%. As shown in Fig. 1 B, 5-aza-2'-deoxycytidine increased the F-positive e-clusters and CFUe colonies in all concentrations used; this result is statistically highly significant $(P<0.001)$. However, there was no correlation between the degree of increment of F-positive colonies and the dose of 5-aza-2'-deoxycytidine used ( $r=0.23$, not significant), suggesting that maximal induction of $\mathrm{Hb} F$ was achieved by the lower concentration of 5-aza-2'-deoxycytidine used in our cultures. Note that the frequency of F-positive colonies was increased even in concentrations of 5-aza-2'-deoxycytidine (1 $\times 10^{-9}-1 \times 10^{-8} \mathrm{M}$ ), which only slightly decreased the number of erythroid colonies (compare Fig. $1, A$ and $B$ ).

Globin biosynthesis was measured in pooled CFUe and e-cluster colonies and separately in BFUe-derived colonies. The $\gamma / \gamma+\beta$ synthetic ratios in control cultures ranged from 0.08 to 0.13 in the CFUe and 0.10 to 0.28 in the BFUe colonies. $\gamma / \gamma+\beta$ ratios were measured in the clonal cultures of cells preexposed to various concentrations of 5-aza-2'-deoxycytidine, and the results in these experimental cultures were expressed as percent of the $\gamma / \gamma+\beta$ ratio of the control cultures done in the absence of 5-aza-2'-deoxycytidine. The data in Fig. $2 A$ show that 5-aza- 2 -deoxycytidine increases $\gamma$-chain biosynthesis in erythroid colonies produced by e-clusters and CFUe in a concentration-related fashion $(r=0.7954 ; P<0.01)$. As shown in Fig. $2 B$, low concentrations of 5-aza-2'-deoxycytidine $\left(5 \times 10^{-9}-1 \times 10^{-9} \mathrm{M}\right)$ do not appear to stimulate $\mathrm{Hb} \mathrm{F}$ synthesis in BFUe-derived colonies. However, preincubation of bone marrow with $1 \times 10^{-8}-5 \times 10^{-8} \mathrm{M} 5$-aza-2'-deoxycytidine increased $\mathrm{Hb} F$ production in six of seven BFUe cultures,

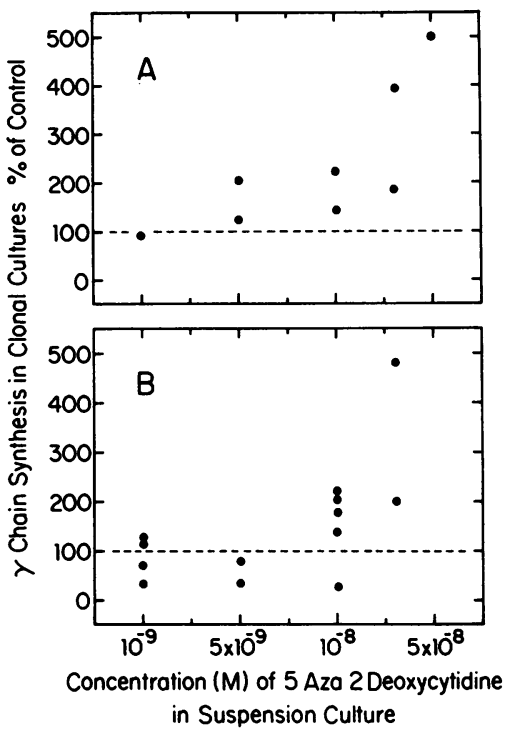

Figure 2. Effect of 5-aza-2'-deoxycytidine on $\mathrm{Hb} \mathrm{F}$ expression. Globin chain biosynthetic measurements done on $(A)$ pooled preparations of CFUe colonies and e-clusters from plasma clot cultures, and $(B)$ BFUe colonies from methylcellulose cultures. $\gamma$ Globin biosynthesis in the experimental cultures is expressed as percent of $\gamma$-globin biosynthesis in control cultures of the same cells done in the absence of 5-aza-2'-deoxycytidine. Culture conditions are as in Fig. 1.

suggesting that the increment of $\mathrm{Hb} \mathrm{F}$ expression is related to the concentration of the drug in the media $(r=0.5649 ; P$ $<0.025$ ).

Bone marrow from a normal person (case B, Table I) was used for the following experiment. The cells were incubated for $42 \mathrm{~h}$ in suspension culture in the presence of $5 \times 10^{-9}$ or 3 $\times 10^{-8} \mathrm{M}$ 5-aza-2'-deoxycytidine, part of the cultured cells was plated in plasma clots, while incubation of another part continued (after new suspension media containing 5-aza-2'-deoxycytidine were added). At $60 \mathrm{~h}$, these latter samples were plated in plasma clots. Measurement of the number of colonies and the frequency of F-positive colonies in the plasma clot cultures allowed an assessment of whether prolongation of the time of exposure of progenitor cells to 5-aza-2'-deoxycytidine during suspension culture increases $\mathrm{Hb} \mathrm{F}$ expression in colonies formed by the so-exposed progenitors. As shown in Fig. 3,

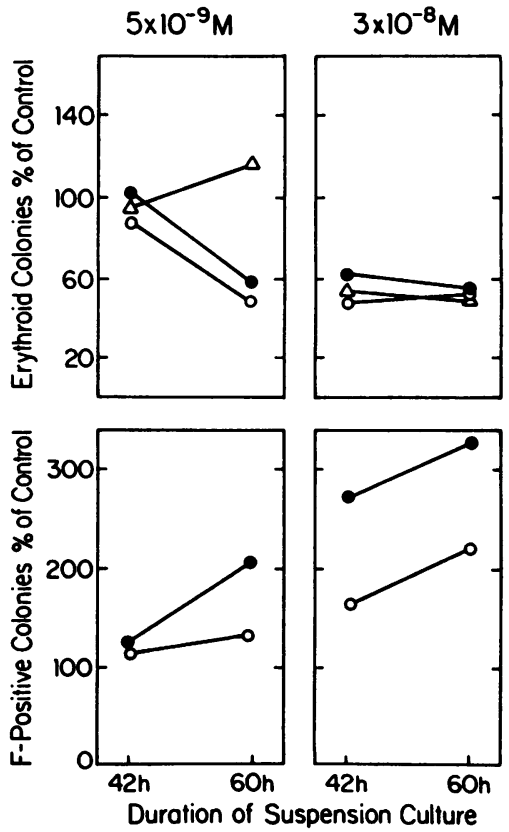

Figure 3. Comparison of the effects of 42-h suspension culture to the effects of a 60 -h suspension culture of bone marrow cells in the presence of 5-aza-2'-deoxycytidine. Controls were cultures of the same cells in the absence of 5-aza-2'-deoxycytidine. (Top) Effects on the clonogenic potential of erythroid progenitors. (Bottom) Effect on $\mathrm{Hb} \mathrm{F}$ expression in erythroid colonies. (๑) e-clusters, (o) CFUe colonies, and $(\Delta)$ BFUe colonies. 
exposure of bone marrow cells to 5-aza-2'-deoxycytidine for 60 $h$ increased $F$-positive colonies more than the exposure to 5-aza-2'-deoxycytidine for $42 \mathrm{~h}$. The increment was higher in the bone marrow cells incubated with $3 \times 10^{-8} \mathrm{M}$ compared with $5 \times 10^{-9}$ M 5-aza-2'-deoxycytidine.

We subsequently tested whether 5-aza-2'-deoxycytidine induces $\mathrm{Hb} \mathrm{F}$ expression in erythroblasts. Bone marrow cells from an $\mathrm{Hb} \mathrm{S}$ homozygote (patient W.H.) were cultured in the presence of $1 \times 10^{-8}$ or $3 \times 10^{-8} \mathrm{M} 5$-aza-2'-deoxycytidine, as well as in the absence of the drug. After $42 \mathrm{~h}$ in suspension culture, erythroblasts were separated by immune adherence, smears were prepared, they were doubly stained with anti- $\gamma$ FITC and anti- $\beta$ rhodamine, and the proportion of F erythroblasts determined. $6 \%$ of the reticulocytes of patient W.H. were F reticulocytes, and $29 \%$ of his red cells were F cells. In the suspension culture done in the absence of 5-aza-2'-deoxycytidine, $22 \%$ of erythroblasts were positive for $\mathrm{Hb} \mathrm{F}$. In the suspension culture done in the presence of $1 \times 10^{-8}$ and $3 \times 10^{-8}$ M 5-aza-2'-deoxycytidine, 36.7 and $44.8 \%$ of the erythroblasts (respectively) were positive for $\mathrm{Hb} \mathrm{F}$.

Effects of Ara-C. Bone marrow samples from normal volunteers were cultured, in suspension, in the absence of Ara-C (controls), or in the presence of various concentrations of this compound (Table I). At the end of the 42-h incubation, the cells were plated in plasma clot and in methylcellulose cultures done in the absence of Ara-C. In these clonal cultures, we assessed whether preincubation of bone marrow cells with Ara-C influenced the clonogenic potentials of progenitors or the $\mathrm{Hb} \mathrm{F}$ expression in erythroid colonies.

Effects of Ara-C on the clonogenic potentials of erythroid progenitors are summarized in Fig. $4 \mathrm{~A}$. Suspension culture of bone marrow cells for $42 \mathrm{~h}$ in the presence of $5 \times 10^{-10} \mathrm{M}$ Ara-C did not decrease the clonogenic potential of erythroid progenitors (indeed, it may have slightly increased growth of e-clusters and CFUe). Erythroid progenitors were, however, reduced when the cells were cultured for $42 \mathrm{~h}$ in the presence of $3 \times 10^{-8}-1 \times 10^{-9} \mathrm{M}$ Ara-C.
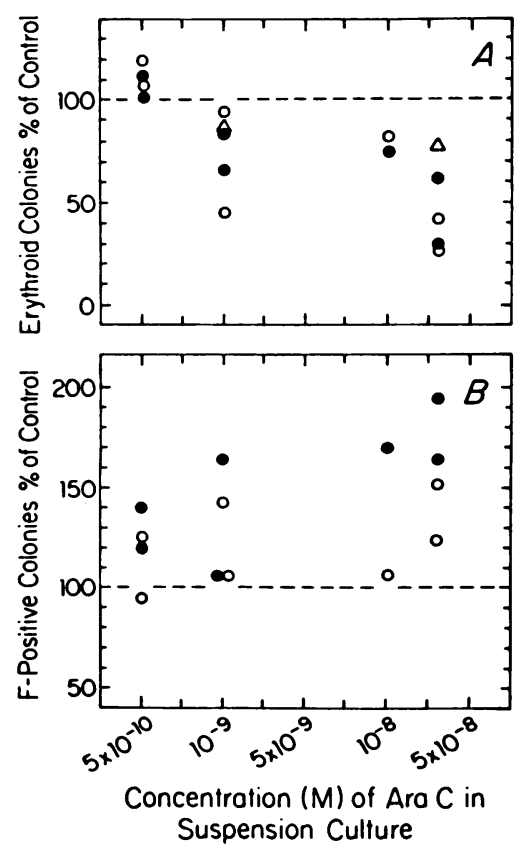

Figure 4. Effect of Ara-C on $(A)$ the clonogenic potential of erythroid progenitors, and $(B) \mathrm{Hb} \mathrm{F}$ expression in erythroid colonies. Data in each experimental culture are expressed as percent of control cultures done in the absence of Ara-C. Findings in (๑) e-clusters, $(0)$ CFUe colonies, and $(\Delta)$ BFUe colonies.
Fig. $4 B$ presents the results of evaluation of $\mathrm{Hb} F$ expression in CFUe colonies and, separately, in e-clusters by immunofluorescence. In control cultures, frequency of F-positive e-clusters ranged from 13.1 to $32 \%$, while the frequency of F-positive CFUe ranged from 36.8 to $49.8 \%$. The frequency of F-positive colonies was higher in the Ara-C preincubated cultures, compared with their controls, in 12 of 14 experiments. However, the stimulation of $\mathrm{Hb} \mathrm{F}$ expression was less pronounced than that observed in the 5-aza-2'-deoxycytidinetreated samples (compare Fig. $4 B$ to Fig. $1 B$ ). Regression analyses showed correlation between increment in F colonies and concentration of Ara-C in the media ( $r=0.7028 ; P$ $<0.0025$ ).

Results of globin biosynthesis in CFUe colonies and eclusters are presented in Fig. $5 A$. In the control cultures, $\gamma / \gamma$ $+\beta$ biosynthetic ratios ranged from 0.05 to 0.13 for CFUe (and e-clusters) and 0.05 to 0.20 for BFUe colonies. In six out of nine experiments, preincubation of bone marrow cells with various concentrations of $\mathrm{Ara}-\mathrm{C}$ increased $\mathrm{Hb} \mathrm{F}$ biosynthesis in CFUe colonies and e-clusters, compared with the values of controls. Regression analyses failed to reveal a statistically significant correlation between $\mathrm{Hb} \mathrm{F}$ synthesis and concentration of Ara-C in the media ( $r=0.3908$, not statistically significant). Ara-C did not influence $\mathrm{Hb} \mathrm{F}$ biosynthesis in BFUe colonies (Fig. $5 B ; r=0.1712$, not statistically significant).

Frequencies of $F$-positive colonies were determined at both 42 and $60 \mathrm{~h}$ after suspension cultures of a normal bone marrow (case B, Table I) in the presence of $1 \times 10^{-9}$ and $3 \times 10^{-8}$ M Ara-C (Fig. 6). This experiment showed that prolongation of suspension culture from 42 to $60 \mathrm{~h}$ increased $\mathrm{F}$ colonies when cultures were done in the presence of $3 \times 10^{-8} \mathrm{M}$ Ara-C, but not in cultures done with $1 \times 10^{-9} \mathrm{M}$ Ara-C.

We also examined whether Ara- $\mathrm{C}$ influences $\mathrm{Hb} \mathrm{F}$ expression in erythroid cells maturing during suspension culture. Homozygous $\mathrm{Hb} \mathrm{S}$ bone marrow cells (from patient W.H.) were cultured in the presence of $1 \times 10^{-8}$ or $3 \times 10^{-8} \mathrm{M}$ Ara-C. At the end of the 42-h incubation, erythroblasts were enriched by immune adherence and stained with anti- $\gamma$ and anti- $\beta$ chain FITC. Both concentrations of Ara-C increased F erythroblasts by $\sim 1$.4-fold, compared with control suspension cultures. Ara-C had a striking effect on morphology of erythroblasts; $70-80 \%$ of erythroblasts were overtly megaloblastic,

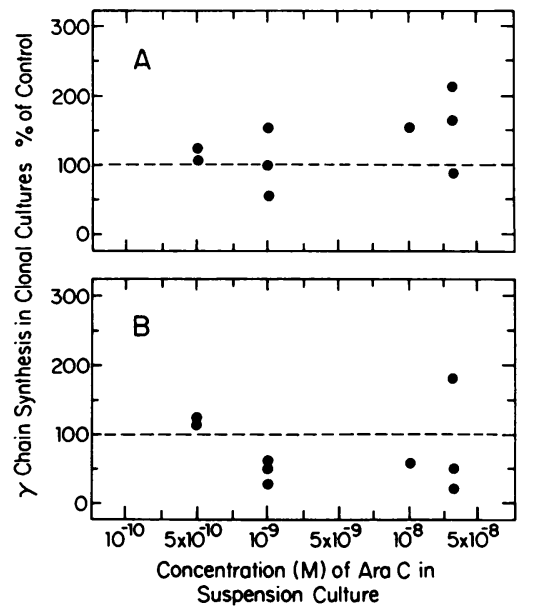

Figure 5. Effect of Ara-C on $\mathrm{Hb} \mathrm{F}$ expression. Globin chain biosynthetic measurements done on $(A)$ pooled preparation of e-clusters and CFUe colonies from plasma clot cultures, and $(B)$ BFUe colonies from methylcellulose cultures. $\gamma$ Globin biosynthesis in each experimental culture is expressed as percent of $\gamma$-globin biosynthesis in control cultures (of the same cells) done in the absence of Ara-C. 


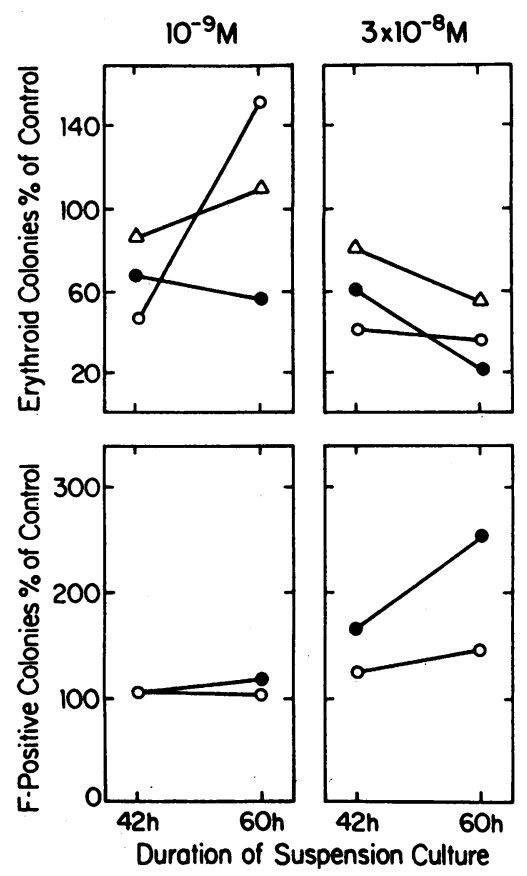

while megaloblastic erythroblasts were rare in the control cultures. In 5-aza-2'-deoxycytidine-treated cultures, $\sim 15 \%$ of the erythroblasts were megaloblastic.

Effect of hydroxyurea. There was no reduction of erythroid progenitor cell numbers when the bone marrow cells were cultured for $42 \mathrm{~h}$ in suspension in the presence of $1 \times 10^{-6}-1$ $\times 10^{-8} \mathrm{M}$ hydroxyurea (data not shown). Slight reduction (20-40\% of control values) was observed when bone marrow cells were preincubated for $42 \mathrm{~h}$ in the presence of $5 \times 10^{-5}-5$ $\times 10^{-6} \mathrm{M}$ hydroxyurea (Fig. $7 \mathrm{~A}$ ). In control cultures, frequencies of F-positive e-clusters and F-positive CFUe ranged from
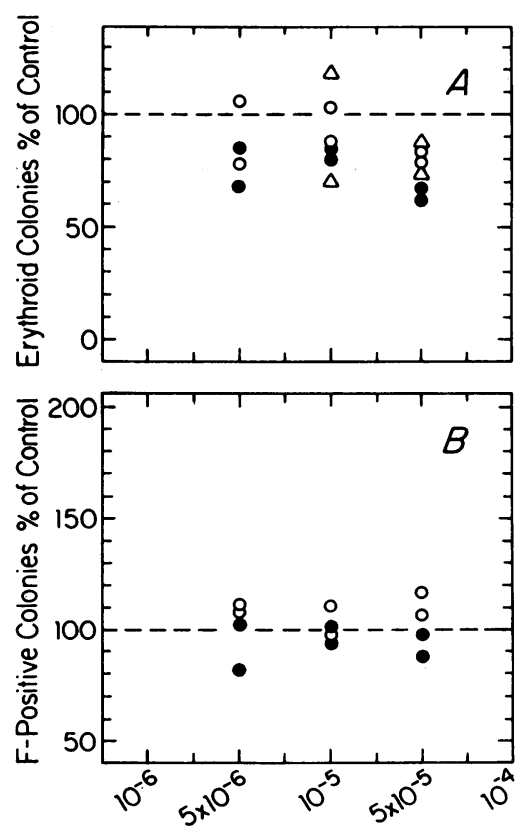

Concentration (M) of Hydroxyurea in Suspension Culture
Figure 6. Comparison of the effects of 42-h the effects of a 60 -h suspension culture of bone marrow cells in the Findings in experimenpressed as percent of values obtained in conabsence of Ara-C. Effects on the clonogenic potential of progenitors. (Bottom) Effects on $\mathrm{Hb} \mathrm{F}$ exprese-clusters, (O) CFU colonies, and $(\Delta)$ BFUe colonies. tal samples are ex-
Figure 7. Effect of hydroxyurea on $(A)$ the clonogenic potential of erythroid progenitors, and $(B) \mathrm{Hb} F$ expression in erythroid colonies. Measurements in experimental samples are expressed as percent of measurements in control cultures done in the absence of hydroxyurea. Findings in (๑) eclusters, (O) CFUe colonies, and $(\Delta)$ BFUe colonies.
14.0 to $24.1 \%$ and 48.3 to $65.5 \%$, respectively. As shown in Fig. $7 B$, exposure, during suspension culture, of erythroid progenitors to $5 \times 10^{-5}-5 \times 10^{-6} \mathrm{M}$ hydroxyurea essentially produced no increment in F-positive e-clusters or CFUe colonies. By globin biosynthesis, 8 of 12 biosynthetic determinations of $\mathrm{Hb} \mathrm{F}$ expression in e-clusters and CFUe colonies showed slightly higher values in the experimental samples compared with controls (Fig. $8 A$ ), but the results were not statistically significant. Measurements in BFUe cultures (Fig. 8 $B$ ) showed that 10 of 18 experimental samples had higher, 4 had the same, and 4 had lower $\gamma / \gamma+\beta$ ratios compared with controls; these differences were not statistically significant.

\section{Discussion}

The mechanisms whereby S-stage compounds stimulate $\mathrm{Hb} \mathrm{F}$ synthesis in vivo remain controversial. In the case of 5-azacytidine, direct induction of $\gamma$-gene expression by the demethylating action of this drug has been proposed (1-7). Studies in baboons (8) and monkeys (14) have shown, however, that at least in part, the induction of $\mathrm{Hb} \mathrm{F}$ by 5 -azacytidine could be explained by the acute-phase regeneration kinetics triggered by the drug treatment. Induction of $\mathrm{Hb} F$ in vivo has also been observed after treatment of patients or animals with other Sstage or M-stage compounds: cytosine arabinoside $(11,13)$, hydroxyurea $(9,13,14,17,26)$, myleran (27), and vinblastin $(12,14)$. In several of these studies, daily measurements of $F$ reticulocyte production have demonstrated that the induction of $F$ cell formation occurs during the stage of erythroid marrow regeneration $(8,11-14)$. Such results are compatible with the hypothesis that the postchemotherapy induction of $F$ cell formation is due to premature commitment of earlier progenitors or to cell cycle changes induced by the rapid erythroid regeneration kinetics that follow the cytoreduction phase of the drug treatment $(10,14)$. This hypothesis, however, has been challenged by other investigators. Humphries et al. (6) reported that there is no consistent suppression of the erythroid marrow in patients treated with 5-azacytidine, suggesting that the cytoreduction and the resultant erythroid regeneration play a minor role in the stimulation of $\mathrm{Hb} F$ by this compound. Platt et al. treated two patients with hydroxyurea and observed induction of $F$ cells in the absence of reduction in absolute reticulocytes (26); however, the changes in other

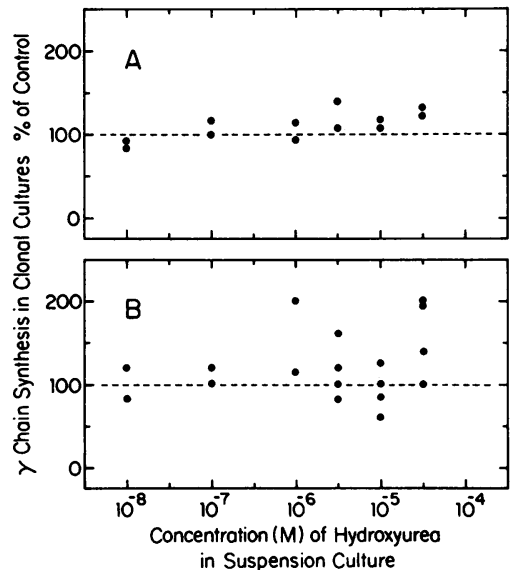

Figure 8. Effect of hydroxyurea on $\mathrm{Hb} \mathrm{F}$ expression. Globin chain biosynthetic measurements done on $(A)$ pooled e-clusters and CFUe from plasma clot cultures, and $(B)$ BFUe colonies from methylcellulose cultures. Expression of results as in legend to Fig. 7. 
parameters (such as white blood cell and platelet counts) indicated that there was bone marrow suppression after the hydroxyurea treatment (26). In the study of Dover et al. (17), eight patients were treated with hydroxyurea, and all responded with cytoreduction, as revealed by the decrease in the values of absolute reticulocytes; these authors observed that $F$ reticulocyte response was higher in two patients having the lowest erythroid suppression, and suggested that a mechanism other than the postcytoreduction fast erythroid regeneration kinetics could underlie the stimulation of $\mathrm{Hb} \mathrm{F}$ by hydroxyurea.

In this study, we tested the influence of three S-stage compounds on erythroid progenitors in vitro by exposing the progenitor cells to these compounds for a period of 42-60 $\mathrm{h}$ and then allowing them to generate colonies in a drug-free environment. Studies of $\mathrm{Hb} \mathrm{F}$ expression in the colonies generated in the clonogenic assays allowed us to obtain information, first, on whether the preexposure of bone marrow cells to an S-stage compound resulted in stimulation of $\mathrm{Hb} F$ in the progeny formed in a drug-free environment; and second, on the type of target cells on which this effect had been exerted. Previous studies have shown that differentiation and maturation of erythroid cells continues in the presence of erythropoietin during suspension culture (28). We have found that the relative proportion of proerythroblasts and basophilic erythroblasts, as well as the number of CFUe, are substantially higher at the end of $42 \mathrm{~h}$ suspension culture (compared with the initial inocula), suggesting de novo generation of both proerythroblasts and CFUe (data not shown). Thus, formation of increased numbers of F-positive erythroblasts at the end of suspension culture suggests an effect of the S-stage compound either on erythroblasts present during inoculation of the culture and/or an effect on late erythroid progenitors (e-cluster-forming cells or late $\mathrm{CFUe}$ ) which generate erythroblasts during the suspension culture time. Increase of $\mathrm{Hb} \mathrm{F}$-positive e-clusters in the clonogenic assay is interpreted to indicate an effect on e-clusterforming cells present originally in the suspension culture or generated from CFUe's during suspension culture. Likewise, induction of $\mathrm{Hb} F$ in CFUe-derived colonies is expected to result from action of the drug on CFUe present originally in the suspension cultures, as well as on in vitro-generated CFUe through BFUe maturation (during the time of suspension culture).

Analyses of $\mathrm{Hb} \mathrm{F}$ expression showed that 5-aza-2'-deoxycytidine had stimulated $\mathrm{Hb} \mathrm{F}$ production in all types of colonies formed (BFUe, CFUe, and e-clusters), as well as in erythroblasts present at the end of the $42-\mathrm{h}$ incubation period. There was increased $\mathrm{Hb} F$ synthesis in CFUe colonies, e-clusters, and erythroblasts, in the Ara-C-treated cultures, but the effects were less pronounced than those of 5-aza-2'-deoxycytidine. There was no stimulation of $\mathrm{Hb} \mathrm{F}$ in hydroxyurea-treated cultures; this negative result, however, may relate to the fact that relatively nontoxic concentrations of hydroxyurea were used.

Since it is known that 5-azacytidine induces the expression of several inactive genes, the in vitro induction of $\mathrm{Hb} \mathrm{F}$ by 5-aza-2'-deoxycytidine can be attributed to demethylation of $\gamma$-globin genes of erythroblasts and progenitors, as previously proposed $(1,2)$. Alternatively, to explain the in vitro induction of $\mathrm{Hb} \mathrm{F}$ by 5 -aza-2'-deoxycytidine, as well as by Ara-C, other mechanisms can be considered. An indirect mechanism involving recruitment of less mature progenitors with an active $\mathrm{Hb} \mathrm{F}$ program has been proposed to account for the induction of $\mathrm{Hb} \mathrm{F}$ by S-stage-specific compounds in vivo (8-14). The same mechanism could account for our in vitro results. It is reasonable to assume that, in the presence of cytotoxic levels of a cell cycle-specific drug in the suspension culture, the most mature, rapidly dividing progenitors (29) will be preferentially killed, while the less frequently dividing immature progenitors will be spared. As a result of such in vitro cell selection, bone marrow samples preincubated with Ara-C (or with 5-azacytidine) will be enriched in noncycling immature erythroid progenitors. If among these progenitor cells the proportion of cells having a program allowing the $\mathrm{Hb} F$ expression is higher, an increased proportion of $\mathrm{F}$ colonies will be formed when these progenitor cells are plated in clonal erythroid cultures. That $\mathrm{Hb} \mathrm{F}$ induction mainly occurs at doses reducing the total number of colonies (Figs. 1, 2, 4, and 5) is compatible with the selection hypothesis.

Observations on the kinetics of $F$ cell formation after a chronic or an acute stimulation of erythropoiesis in patients or in anemic baboons suggest that rapid erythroid regeneration kinetics underlie the stimulated F cell formation in these conditions (10). Rapid erythroid regeneration may stimulate F cell formation by increasing the chance of premature terminal commitment of earlier cells having an active $\mathrm{Hb} F$ program $(10,30)$. An alternative hypothesis is that the accelerated cycling, per se, activates the fetal globin program in progenitors and precursors. This hypothesis, detailed before (10), assumes that shortening of the erythroid cell cycle below a critical time increases the chance of $\gamma$-gene activation and, hence, $F$ cell formation. This hypothesis could also explain the induction of $\mathrm{Hb} \mathrm{F}$ by cell cycle-specific drugs in vitro. We assume that erythroid progenitors and precursors that incorporate sublethal concentrations of S-stage compounds become temporarily arrested at the $G_{1} / S$ boundary or early S-phase, as other studies of the effect of S-stage compounds on proliferating cells suggest (31). When the drug-treated bone marrow cells are replated in a drug-free environment, some of the surviving and partially synchronized erythroid progenitors are released from their temporary arrest and begin to proliferate with a shortened cycling rate, which may activate the expression the $\gamma$ genes. Increased plating efficiency of leukemic cells exposed previously to sublethal doses of 5-azacytidine (32) can be cited in favor of changes in cell kinetics postdrug treatment.

In summary, the in vitro data that we have generated complement in vivo observations and provide arguments for considering more than one mechanism of induction of $\mathrm{Hb} F$ production by cell cycle-specific drugs.

\section{Acknowledgments}

We thank Betty Nakamoto and Sumiko Kurachi for excellent technical assistance, and Doreen Loomans for typing the manuscript.

This work was supported by grant HL-20899 from National Institutes of Health.

\section{References}

1. DeSimone, J., P. Heller, L. Hall, and D. Swiers. 1982. 5-azacytidine stimulates fetal hemoglobin synthesis in anemic baboons. Proc. Natl. Acad. Sci. USA. 79:4428-4431.

2. Ley, T. J., J. DeSimone, N. P. Anagnou, G. H. Keller, R. K. Humphries, P. H. Turner, N. S. Young, P. Heller, and A. W. Nienhuis. 
1982. 5-azacytidine selectively increases $\gamma$-globin synthesis in a patient with $\beta^{+}$-thalassemia. N. Engl. J. Med. 307:1469-1475.

3. Ley, T. J., J. DeSimone, T. Noguchi, P. H. Turner, A. N. Schechter, P. Heller, and A. W. Nienhuis. 1983. 5-azacytidine increases $\gamma$-globin synthesis and reduces the proportion of dense cells in patients with sickle cell anemia. Blood. 62:370-380.

4. Nienhuis, A. W., T. J. Ley, R. K. Humphries, N. S. Young, and G. Dover. 1985. Pharmacological manipulation of fetal hemoglobin synthesis in patients with severe $\beta$-thalassemia. Ann. NY Acad. Sci. 445:198-211.

5. Charache, S., G. Dover, K. Smith, C. C. Talbot, Jr., M. Moyer, and S. Boyer. 1983. Treatment of sickle cell anemia with 5-azacytidine results in increased fetal hemoglobin production and is associated with nonrandom hypomethylation of DNA around the $\gamma$ - $\delta$ - $\beta$-globin gene complex. Proc. Natl. Acad. Sci. USA. 4842-4846.

6. Humphries, R. K., G. Dover, N. W. Young, J. G. Moore, S. Charache, T. Ley, and A. W. Nienhuis. 1985. 5-Azacytidine acts directly on both erythroid precursors and progenitors to increase production of fetal hemoglobin. J. Clin. Invest. 75:547-557.

7. Dover, G. J., S. Charache, S. H. Boyer, G. Vogelsang, and M. Moyer. 1985. 5-azacytidine increases Hb F production and reduces anemia in sickle cell disease: dose-response analysis of subcutaneous and oral dosage regimens. Blood. 66:527-532.

8. Torrealba de Ron, A., Th. Papayannopoulou, M. S. Knapp, M. Fu, G. Knitter, and G. Stamatoyannopoulos. 1984. Perturbations in the erythroid marrow progenitor cell pools may play a role in the augmentation of $\mathrm{Hb} \mathrm{F}$ by 5-azacytidine. Blood. 63:201-210.

9. Letvin, N. L., D. C. Linch, G. P. Beardsley, K. W. McIntyre, and D. G. Nathan. 1984. Augmentation of fetal hemoglobin production in anemic monkeys by hydroxyurea. $N$. Engl. J. Med. 310:869-873.

10. Stamatoyannopoulos, G., R. Veith, R. Galanello, and Th. Papayannopoulou. 1985. Hb F production in stressed erythropoiesis: observations and kinetic models. Ann. NY Acad. Sci. 445:188-197.

11. Papayannopoulou, Th., A. Torrealba de Ron, R. Veith, G. Knitter, and G. Stamatoyannopoulos. 1984. Arabinosylcytosine induces fetal hemoglobin in baboons by perturbing erythroid cell differentiation kinetics. Science (Wash. DC). 224:617-619.

12. Veith, R., Th. Papayannopoulou, S. Kurachi, and G. Stamatoyannopoulos. 1985. Treatment of baboon with vinblastin: insights into the mechanisms of pharmacologic stimulation of $\mathrm{Hb} \mathrm{F}$ in the adult. Blood. 66:456-459.

13. Veith, R., R. Galanello, Th. Papayannopoulou, and G. Stamatoyannopoulos. 1986. Stimulation of F-cell production in patients with sickle cell anemia treated with cytarabine or hydroxyurea. $N$. Engl. J. Med. 313:1571-1575.

14. Letvin, N. L., D. C. Linch, G. P. Beardsley, K. W. McIntyre, G. Miller, and D. G. Nathan. 1986. Influence of cell cycle phase-specific agents on simian fetal hemoglobin synthesis. J. Clin. Invest. 75:19992005.

15. Dover, G. J., S. H. Boyer, and W. H. Zinkham. 1979. Production of erythrocytes that contain fetal hemoglobin in anemia. J. Clin. Invest. 63:173-176.

16. Papayannopoulou, Th., E. Vichinsky, and G. Stamatoyannopoulos. 1980. Fetal $\mathrm{Hb}$ production during acute erythroid expansion. I. Observations in patients with transient erythroblastopenia and postphlebotomy. Br. J. Haematol. 44:535-546.

17. Dover, G. J., R. K. Humphries, J. G. Moore, T. J. Ley, N. S. Young, S. Charache, and A. W. Nienhuis. 1986. Hydroxyurea induction of hemoglobin $F$ production in sickle cell disease: relationship between cytotoxicity and F-cell production. Blood. 67:735-738.

18. Momparler, R. L., F. L. Momparler, and J. Samson. 1984. Comparison of the antileukemic activity of 5-aza-2'-deoxycytidine,
1- $\beta$-D-arabinofuranosylcytonine and 5-azacytidine against L-1210 leukemia. Leuk. Res. 8:1043-1049.

19. Cooper, K. D., K. Kang, T. L. Bush, and J. M. Hanifin. 1984. Direct depletion and purification of monoclonal antibody defined cells from unfractionated human mononuclear leukocytes using antibody coated polystyrene petri dishes. J. Clin. Lab. Immunol. 13:97-100.

20. Yokochi, T., M. Brice, P. S. Rabinovitch, Th. Papayannopoulou, and G. Stamatoyannopoulos. 1984. Monoclonal antibodies detecting antigenic determinants with restricted expression on erythroid cells: from the erythroid committed progenitor level to the mature erythroblast. Blood. 63:1376-1384.

21. Papayannopoulou, Th., T. Kalmantis, and G. Stamatoyannopoulos. 1979. Cellular regulation of hemoglobin switching: evidence for inverse relationship between fetal hemoglobin synthesis and degree of maturity of human erythroid cells. Proc. Natl. Acad. Sci. USA. 76:6420-6424.

22. Papayannopoulou, Th., M. Brice, and G. Stamatoyannopoulos. 1977. Hemoglobin $F$ synthesis in vitro: evidence for control at the level of primitive erythroid stem cells. Proc. Natl. Acad. Sci. USA. 74:2923-2927.

23. Bigbee, W. L., E. W. Branscomb, H. B. Weintraub, Th. Papayannopoulou, and G. Stamatoyannopoulos. 1981. Cell sorter immunofluorescence detection of human erythrocytes labeled in suspension with antibodies specific for hemoglobin S and C. J. Immunol. Methods. 45:117-127.

24. Stamatoyannopoulos, G., P. E. Nute, Th. Papayannopoulou, T. McGuire, G. Lim, H. F. Bunn, and D. Rucknagel. 1980. Development of a somatic mutation screening system using $\mathrm{Hb}$ Mutants. IV. Successful detection of red cells containing the human frameshift mutants $\mathrm{Hb}$ Wayne and $\mathrm{Hb}$ Cranston using monospecific fluorescent antibodies. Am. J. Hum. Genet. 32:484-496.

25. Righetti, P. G., E. Gianazza, A. M. Gianni, P. Comi, B. Giglioni, S. Ottolenghi, C. Sechi, and C. Rossi-Bernardi. 1979. Human globin chain separation by isoelectric focusing. J. Biochem. Biophys. Methods. 1:45-46.

26. Platt, O. S., S. H. Orkin, G. Dover, G. P. Beardsley, B. Miller, and D. G. Nathan. 1984. Hydroxyurea enhances fetal hemoglobin production in sickle cell anemia. J. Clin. Invest. 74:652-656.

27. Liang, C. C., D. P. Liu, P. C. Jia, Z. H. Ao, S. S. Chen, and K. G. Yang. 1987. Augmentation of fetal hemoglobin in anemic monkeys by meleran. In Developmental Control of Globin Gene Expression. G. Stamatoyannopoulos and A. W. Nienhuis, editors. Alan R. Liss, Inc., New York. pp. 467-477.

28. Dessypris, E. N., and S. B. Krantz. 1984. Effect of pure erythropoietin on DNA synthesis by day 15-human marrow BFUe in shortterm liquid culture. Br. J. Haematol. 56:295-306.

29. Gregory, C. J., and A. C. Eaves. 1978. Three stages of erythropoietic progenitor cell differentiation distinguished by a number of physical and biologic properties. Blood. 51:527-537.

30. Stamatoyannopoulos, G., and Th. Papayannopoulou. 1979. Fetal hemoglobin and the erythroid stem cell differentiation process. In Cellular and Molecular Regulation of Hemoglobin Switching. G. Stamatoyannopoulos and A. W. Nienhuis, editors. Grune \& Stratton Inc., New York. 323-350.

31. Ishikura, H., T. Okazaki, T. Mochizuki, Y. Izumi, M. Tashima, H. Sawada, and H. Uchino. 1985. Effect of antimetabolites and thymidine blockage on the induction of differentiation of HL-60 cells by retinoic acid or $1 \alpha, 25$-dihydroxyvitamin $\mathrm{D}_{3}$. Exp. Hematol. 13:981988.

32. Motoji, T., T. Hoang, D. Tritchler, and E. A. McCulloch. 1985. The effect of 5-azacytidine and its analogues on blast cell renewal in acute myeloblastic leukemia. Blood. 65:894-901. 